\title{
Nachweis der Spiele des Turniers.
}

Anderssen.

Erster Gang: XI., XII., XIII. - Z weiter Gang: I., II., III., IV., V., VI. - Dritter Gang VIII., IX., X., XI., XII. - Vierter Bird.

Gang: I., II., III., IV., V., VI., VII.

Brodie.

Erster Gang: III., IV., V., VI.

Erster Gang: VII., VIII.

Horwitz.

Erster Gang: III., IV., V., VI. - Zweiter Gang: XV., XVI., XVII., XVIII., XIX., XX., XXI. - Dritter Gang: XVII., XVIII., XIX., XX.

Capt. Kennedy.

Erster Gang: XIX., XX. - Z weiter Gang: VII., VIII, IX, X., XI., XII., XIII., XIV. -- Dritter Gang: XIII., XIV., XV., XVI. Vierter Gang: XVI, XVII., XVIII., XIX., XX.

E. S. Kennedy.

Erster Gang: IX., X.

Kieseritzky.

Erster Gang: XI., XII., XIII.

L.öwenthal.

Erster Gang: XIV., XV., XVI.

Lowe.

Erster Gang: I., II.

Majet.

Erster Gang: XIX., XX.

Hucklow.

Erster Gang: IX., X. - Zweiter Gang: XXII., XXIII., XXIV., Newham.

XXV. - Dritter Gang: XIII., XIV., XV., XVI.

\section{Staunton.}

Erster Gang: XVII., XVIII.

Erster Gang: VII., VIII. - Z weiter Gang: XV., XVI., XVII, XVIII, XIX., XX., XXI. - Dritler Gang: VIII., IX., X., XI., XII. Szèn.

- Vierter Gang: VIII., IX., X., XI., XII., XIII., XIV., XV.

Erster Gang: XVII., XVIII. - Z Z weiter Gang: I., II., III., IV., V., VI. - Dritter Gang: XVII., XVIII., XIX., XX. - Vierter Gang: XVI., XVII., XVIII., XIX., XX.

Williams.

Erster Gang: XIV., XV., XVI. - Zweiter Gang: XXII., XXIII., XXIV., XXV. - Dritter Gang: I., II., III., IV., V., VI., VII. Wyvill.

Vierter Gang: VIII., IX., X., XI., XII., XIII., XIV., XV.

Erster Gang: I., II. - Zweiter Gang: VII., VIII., IX., X., XI., XII., XIII., XIV. - Dritter Gang: I., II., III., IV., V., VI., VII. - Vierter Gang: I., II., III., IV., V., VI., VII. 